\title{
PLCG2 is associated with the inflammatory response and is induced by amyloid plaques in Alzheimer's disease
}

\author{
Andy P. Tsai ${ }^{1 \dagger}$, Chuanpeng Dong ${ }^{2 \dagger}$, Peter Bor-Chian Lin ${ }^{1}$, Evan J. Messenger ${ }^{1}$, Brad T. Casali ${ }^{1,3}$, \\ Miguel Moutinho', Yunlong Liu' ${ }^{2}$, Adrian L. Oblak ${ }^{1,4}$, Bruce T. Lamb ${ }^{1,5}$, Gary E. Landreth ${ }^{1,6}$, \\ Stephanie J. Bissel ${ }^{1,5^{*}}$ and Kwangsik Nho ${ }^{4^{*}}$
}

\begin{abstract}
Background: Alzheimer's disease (AD) is characterized by robust microgliosis and phenotypic changes that accompany disease pathogenesis. Accumulating evidence from genetic studies suggests the importance of phospholipase C Y 2 (PLCG2) in late-onset AD (LOAD) pathophysiology. However, the role of PLCG2 in AD is still poorly understood.

Methods: Using bulk RNA-Seq $(N=1249)$ data from the Accelerating Medicines Partnership-Alzheimer's Disease Consortium (AMP-AD), we investigated whether PLCG2 expression increased in the brains of LOAD patients. We also evaluated the relationship between PLCG2 expression levels, amyloid plaque density, and expression levels of microglia specific markers (AIF1 and TMEM119). Finally, we investigated the longitudinal changes of PLCG2 expression in the 5xFAD mouse model of AD. To further understand the role of PLCG2 in different signaling pathways, differential gene expression and co-expression network analyses were performed using bulk RNA-Seq and microglial single-cell RNASeq data. To substantiate the human analyses, we performed differential gene expression analysis on wild-type (WT) and inactivated P/cg2 mice and used immunostaining to determine if the differentially expressed genes/pathways were altered by microglial cell coverage or morphology.

Results: We observed significant upregulation of PLCG2 expression in three brain regions of LOAD patients and significant positive correlation of PLCG2 expression with amyloid plaque density. These findings in the human brain were validated in the 5xFAD amyloid mouse model, which showed disease progression-dependent increases in Plcg 2 expression associated with amyloid pathology. Of note, increased Plcg2 expression levels in 5xFAD mice were abolished by reducing microglia. Furthermore, using bulk RNA-Seq data, we performed differential expression analysis by comparing cognitively normal older adults (CN) with 75th percentile (high) and 25th percentile (low) PLCG2 gene expression levels to identify pathways related to inflammation and the inflammatory response. The findings in the human brain were validated by differential expression analyses between WT and plcg2 inactivated mice. PLCG2 coexpression network analysis of microglial single-cell RNA-Seq data identified pathways related to the inflammatory response including regulation of I-kappaB/NF-kappa B signaling and response to lipopolysaccharide.
\end{abstract}

\footnotetext{
*Correspondence: sbissel@iu.edu; knho@iupui.edu

${ }^{\dagger}$ Andy P. Tsai and Chuanpeng Dong contributed equally to this work.

${ }^{4}$ Department of Radiology \& Imaging Sciences, Indiana University School

of Medicine, Indianapolis, IN, USA

${ }^{5}$ Department of Medical and Molecular Genetics, Indiana University

School of Medicine, Indianapolis, IN, USA

Full list of author information is available at the end of the article
} original author(s) and the source, provide a link to the Creative Commons licence, and indicate if changes were made. The images or other third party material in this article are included in the article's Creative Commons licence, unless indicated otherwise in a credit line to the material. If material is not included in the article's Creative Commons licence and your intended use is not permitted by statutory regulation or exceeds the permitted use, you will need to obtain permission directly from the copyright holder. To view a copy of this licence, visit http://creativecommons.org/licenses/by/4.0/. The Creative Commons Public Domain Dedication waiver (http://creativeco mmons.org/publicdomain/zero/1.0/) applies to the data made available in this article, unless otherwise stated in a credit line to the data. 
Conclusions: Our results provide further evidence that PLCG2 plays an important role in AD pathophysiology and may be a potential target for microglia-targeted AD therapies.

Keywords: Alzheimer's disease, Microglia, PLCG2, Inflammatory response, Co-expression network analysis, Single-cell RNA-Seq analysis

\section{Background}

Late-onset Alzheimer's disease (LOAD) is the most common form of Alzheimer's disease (AD), with symptoms arising after age 65 [1]. Age, sex, and the apolipoprotein $\varepsilon 4$ allele ( $A P O E \varepsilon 4)$ are the three greatest risk factors for LOAD [2-4]. LOAD risk doubles every 5 years after age 65 , and over $60 \%$ of AD patients are female [2, 5]. Recent large-scale genome-wide association studies (GWAS) have identified more than 30 genetic loci associated with LOAD, including $A P O E$, which is a well-established genetic risk factor $[6,7]$, but the mechanisms underlying the development of LOAD are incompletely understood.

Importantly, approximately $40 \%$ of the susceptibility genes identified in genetic studies of LOAD are immune- and microglia-related, suggesting that microglia are involved in modulating $\mathrm{AD}$ pathology [8]. To date, it is known that phospholipase $\mathrm{C} \gamma 2$ (PLCG2) might be important in $\mathrm{AD}$ due to the pervious findings that a hypermorphic variant in PLCG2, rs72824905, is protective against $\mathrm{AD}$ risk [9-11]. However, the role of PLCG2 has not yet been comprehensively explored. PLCG2 is a membrane-associated enzyme that catalyzes the conversion of phospholipid PIP2 (1-phosphatidyl-1D-myoinositol 4,5-bisphosphate) to IP3 (1D-myo-inositol 1,4,5-trisphosphate) and DAG (diacylglycerol), which plays a crucial role in cell-surface receptor signal transduction [12]. In AD, the effect of PLCG2 might depend on the stage of neurodegeneration, the gene expression, the enzymatic activity, and the formation of intracellular signaling complexes in microglia [10, 13-16]. Therefore, a better understanding of the role of PLCG2 in AD and microglia is needed for the development of PLCG2directed therapeutics.

Here, we performed differential gene expression and gene-set enrichment analysis of transcriptomic data, including single-cell RNA-Seq data, from human and mouse brain regions to investigate the role of PLCG2 in AD. Expression levels of PLCG2 were increased in LOAD compared to those in cognitively normal older adults $(\mathrm{CN})$ and were positively associated with both amyloid plaques and expression levels of microglial marker genes. Similarly, in a 5xFAD mouse model, Plcg2 expression increased in a disease progression-dependent manner and Plcg2 was highly expressed in plaque-induced microglia in the brain. Importantly, differential expression analysis in $\mathrm{CN}$ and a Plcg2-inactivated mouse model identified inflammation-related pathways, suggesting a potential role for PLCG2 in inflammation-related pathways. Notably, the differentially expressed genes/pathways were not altered by microglial cell coverage or morphology, PLCG2 co-expression network analysis of microglial single-cell RNA-Seq data identified pathways related to the inflammatory response, including regulation of I-kappaB/NF-kappa B signaling, and response to lipopolysaccharide.

Overall, our results suggest that PLCG2 plays an important role in the inflammation-related pathway in $\mathrm{AD}$ and that PLCG2 might be a microglia-associated potential therapeutic target in $\mathrm{AD}$ treatment.

\section{Methods \\ Bulk RNA-Seq data analysis in the post-mortem human brain}

RNA-Seq data were downloaded from the Accelerating Medicines Partnership for Alzheimer's Disease (AMPAD) Consortium (https://adknowledgeportal.synapse. org/Explore/Programs/DetailsPage?Program=AMP-AD) through the Synapse database (https://www.synapse. org/): the Mayo Clinic Brain Bank cohort (Mayo; https:// www.synapse.org/\#!Synapse:syn8466812), the Mount Sinai Medical Center Brain Bank (MSBB; https://www. synapse.org/\#!Synapse:syn8484987) cohort, and the Religious Orders Study and Memory and Aging Project (ROS/MAP; https://www.synapse.org/\#!Synapse:syn84 56629) cohort (Table 1) [17-19].

Table 1 shows the demographic information of the participants included in this study

\begin{tabular}{|c|c|c|c|c|c|c|c|c|c|c|c|c|c|c|c|c|c|c|}
\hline \multirow{2}{*}{$\begin{array}{l}\text { Brain region } \\
\text { Tissue cohort } \\
\text { Diagnosis }\end{array}$} & \multicolumn{2}{|c|}{$\begin{array}{c}\text { Temporal Cortex } \\
\text { (Mayo) }\end{array}$} & \multicolumn{3}{|c|}{$\begin{array}{c}\text { Parahippocampal Gyrus } \\
\text { (MSBB) }\end{array}$} & \multicolumn{3}{|c|}{$\begin{array}{c}\text { Superior Temporal Gyrus } \\
\text { (MSBB) }\end{array}$} & \multicolumn{3}{|c|}{$\begin{array}{l}\text { Inferior Frontal Gyrus } \\
\text { (MSBB) }\end{array}$} & \multicolumn{3}{|c|}{$\begin{array}{c}\text { Frontal Pole } \\
\text { (MSBB) }\end{array}$} & \multicolumn{2}{|c|}{$\begin{array}{c}\text { Cerebellum } \\
\text { (Mayo) }\end{array}$} & \multicolumn{2}{|c|}{$\begin{array}{c}\text { Dosolateral Prefrontal Cortex } \\
\text { (ROSMAP) }\end{array}$} \\
\hline & $\mathrm{CN}$ & LOAD & $\mathrm{CN}$ & $\mathrm{MCl}$ & LOAD & $\mathrm{CN}$ & $\mathrm{MCl}$ & LOAD & $\mathrm{CN}$ & $\mathrm{MCl}$ & LOAD & $\mathrm{CN}$ & $\mathrm{MCl}$ & LOAD & $\mathrm{CN}$ & $A D$ & $\mathrm{CN}$ & Probable AD \\
\hline No. of participar & 71 & 80 & 16 & . & 78 & & 18 & 98 & & Tore & & & & & & & & \\
\hline Sex (Female/Ma & $35 / 36$ & $49 / 31$ & $11 / 5$ & $7 \pi$ & $53 / 25$ & $16 / 5$ & $11 / 7$ & $65 / 33$ & $12 / 6$ & $7 / 9$ & 69 & $16 / 6$ & $11 / 9$ & $75 / 36$ & & $47 / 32$ & $121 / 77$ & $149 / 66$ \\
\hline Mean age at death (SD), years & $82.7(8.5)$ & $82.6(7.7)$ & $83.5(8.9)$ & $80.6(10.8)$ & $85.5(6.1)$ & $33.8(8.1)$ & $82.1(10.4)$ & $84.5(6.7)$ & $83.2(8.6)$ & $0.8(10.5)$ & $85.4(6.0)$ & $83.1(7.7)$ & $83.2(9.8)$ & $85.4(5.9)$ & $82.3(8.3)$ & $82.5(7.7)$ & $84.9(5.2)$ & $88.1(3.2)$ \\
\hline APOE genotype $(\varepsilon 4+\varepsilon \varepsilon 4-)$ & $62 / 9$ & $38 / 42$ & $14 / 2$ & 9/5 & $57 / 21$ & $17 / 4$ & $12 / 6$ & $65 / 33$ & $\begin{array}{l}16 / 2 \\
\end{array}$ & $8 / 8$ & $72 / 30$ & $19 / 3$ & $13 / 7$ & $70 / 41$ & $62 / 10$ & $38 / 41$ & $31 / 167$ & $82 / 133$ \\
\hline
\end{tabular}


In the Mayo Clinic RNA-Seq dataset, the RNA-seqbased whole transcriptome data were generated from the temporal cortex and cerebellum. In the MSBB dataset, data were generated from parahippocampal gyrus, inferior frontal gyrus, superior temporal gyrus, and frontal pole. In the ROSMAP dataset, the RNA-seq data were generated from dorsolateral prefrontal cortices. The procedures for sample collection, post-mortem sample descriptions, tissue and RNA preparation methods, library preparation and sequencing methods, and sample quality controls were previously described in detail [18-22].

To investigate the diagnostic group difference in PLCG2 expression among $\mathrm{CN}, \mathrm{MCI}$, and those with LOAD, we used the limma software [23] to perform differential expression analysis [21]. Age, sex, and APOE $\varepsilon 4$ carrier status were used as covariates. To investigate the relationship between PLCG2 expression levels and amyloid plaque density (number of plaques $/ \mathrm{mm}^{2}$ ) [19, 22] and expression levels of microglia-specific markers (AIF1 and TMEM119), we used linear regression models with PLCG2 expression levels as a dependent variable and plaque density or microglial-specific markers and age, sex, and $A P O E \& 4$ carrier status as explanatory variables. The regression was performed with the "glm" function of the stats package in $\mathrm{R}$ (version 3.6.1).

The expression levels of PLCG2, AIF1, and TMEM119 were downloaded from the Synapse database, and the $\log 2$ counts-per-million $(\log C P M)$ normalized data of RNA-seq were used for the following analysis in this study.

\section{Microglia single-cell RNA-Seq data analysis}

Single-cell transcriptomic data from human microglia isolated from fresh autopsy tissue were download from the AMP-AD consortium database, for which the raw data are accessible through Synapse (https://www.synap se.org/\#!Synapse:syn21438358) [24]. Brain myeloid cells isolated from the dorsolateral prefrontal cortex of 12 donors (four mild cognitive impairment (MCI) and eight AD patients) of both sexes from the ROS/MAP cohort were used. Data were processed with cell ranger count (CellRanger version 5.0.1) [24] using a custom reference package based on the human reference genome GRCh38 (Gencode.V32). Subsequent data analysis was carried out using the Seurat package (v 4.0.0) [25]. We further filtered out cells by genes detected per cell (nFeature_RNA) based on whether over 5000 or less than 200 were detected. Dead cells were excluded by retaining cells with less than $5 \%$ mitochondrial reads (percent. $m t<5 \%$ ). A total of 15,021 cells were qualified for the following analysis.
Microglia single-cell RNA-Seq analyses were performed by Seurat package in R [25]. Data were normalized using the NormalizeData() function, and the variable features were identified using the FindVariableFeatures() with 2000 genes; the selection method was set to "vst," a variance-stabilizing transformation. To identify the integration of anchor genes among the 12 samples, the FindIntegrationAnchors() function was used with 20 principal components and 2000 genes. Using Seurat's Integrate$\mathrm{Data}()$ function, samples were combined into one object. The data were scaled using the ScaleData() function to reduce the dimensionality of this dataset; principal component analysis was used, and the first 30 principal components were summarized further using UMAP dimensionality reduction. Clustering was conducted using the FindNeighbors() and FindClusters() functions using 30 principal components and a resolution parameter set to 0.6. Due to the sparse nature of single-cell transcriptomic profiles, an ensemble tree-based algorithm, GENIE3, was used to reconstruct the co-expression module networks of PLCG2 [26], which calculates the coexpression pattern of PLCG2 and the expression pattern of the other 2469 genes that are expressed in more than $10 \%$ of the total number of cells.

\section{Differential gene expression and pathway enrichment analysis}

The limma package in R software was used to identify differentially expressed genes. The ClusterProfiler package was used to automate biological-term classification and enrichment analysis for the upregulated and downregulated genes [27].

\section{Mice}

Wild-type (WT) and 5xFAD mice used for immunofluorescence and GPCR studies were maintained on the C57BL/6J background and purchased from the Jackson Laboratory (JAX MMRRC Stock\# 034848). One-, 4-, 6-, and 8-month-old mice were used. In the PLX5622 animal treatment study described in the "Methods" section below, we used WT and 5xFAD mice that were maintained as mixed C57BL/6 6 and SJL background 5xFAD mice [B6SJL-Tg (APPSwFlLon, PSEN1*M146L"L286V) 6799Vas, Stock \#34840-JAX]). The 5xFAD transgenic mice overexpress the following five FAD mutations: the APP (695) transgene containing the Swedish (K670N, M671L), Florida (I716V), and London (V7171) mutations, and the PSEN1 transgene containing the M146L and L286V FAD mutations.

To deplete microglia, both WT and 5xFAD mice were treated PLX5622, a colony-stimulating factor receptor-1 antagonist, at 4 months of age. Either normal rodent diet or PLX5622-containing chow was administered for 
28 days. An additional cohort of 4-month-old mice was treated with PLX5622 or the control diet for 28 days, then those initially administered the PLX5622 feed were given a normal rodent diet for an additional 28 days [28]. At 6 months of age, this cohort of mice was euthanized. PLX5622 was provided by Plexxikon formulated as the AIN-7 diet at $1200 \mathrm{mg} / \mathrm{kg}$.

Plcg2 inactivation (PLCG2 inact) mice for differential expression analysis were generated by IU/JAX/ UCI MODEL-AD consortium and maintained on the C57BL/6J background (JAX MMRRC Stock\# 029910; https://www.model-ad.org/strain-table/). PLCG2 inact mice were generated by using CRISPR/cas9 endonuclease mediated genome editing to introduce a 13 bp knockout mutation (nucleotides 1570 to 1582).

Up to five mice were housed per cage with SaniChip bedding and LabDiet ${ }^{\circledR} 5 \mathrm{~K} 52 / 5 \mathrm{~K} 67$ (6\% fat) feed. The colony room was kept on a 12:12-h light/dark schedule with the lights on from 7:00 am to 7:00 pm daily. The mice were bred and housed in specific-pathogen-free conditions. Both male and female mice were used, and the numbers of male and female mice were equally distributed. The number of mice used for each experiment is stated in the corresponding figure legends, and the results of individual values are shown in the scatter plot.

Mice were euthanized by perfusion with ice-cold phosphate-buffered saline (PBS) following full anesthetization with Tribromoethanol [Avertin ${ }^{\circledR}$ ] (125-250 mg/kg intraperitoneal injection). Animals used in the study were housed in the Stark Neurosciences Research Institute Laboratory Animal Resource Center, Indiana University School of Medicine. All animals were maintained, and experiments performed in accordance with the recommendations in the Guide for the Care and Use of Laboratory Animals of the National Institutes of Health. The protocol was approved by the Institutional Animal Care and Use Committee (IACUC) at Indiana University School of Medicine.

\section{Mouse RNA isolation for qPCR and NanoString nCounter analysis}

The cortical and hippocampal regions were micro-dissected and stored at $-80^{\circ} \mathrm{C}$. The frozen brain tissue was homogenized in buffer consisting of $20 \mathrm{mM}$ Tris- $\mathrm{HCl}$ $(\mathrm{pH}=7.4), 250 \mathrm{mM}$ sucrose, $0.5 \mathrm{mM}$ ethylene glycol-bis ( $\beta$-aminoethyl ether)-N,N,N,N'-tetraacetic acid (EGTA), $0.5 \mathrm{mM}$ ethylenediaminetetraacetic acid (EDTA), and RNase-free water and were stored in an equal volume of RNA-Bee (Amsbio, CS-104B) at $-80^{\circ} \mathrm{C}$ until RNA extraction was performed. RNA was isolated by chloroform extraction and purified using the Purelink RNA Mini Kit (Life Technologies). The cDNA was prepared from 750 ng of RNA using the High-Capacity of RNA-to-cDNA kit
(Applied Biosystems), and qPCR was performed on the StepOne Plus Real-Time PCR system (Life Technologies) with the Taqman Gene Expression Assay (Mm01242530_ $\mathrm{m} 1$, Life Technologies). Relative gene expression was determined with the $\triangle \triangle \mathrm{CT}$ method and was assessed relative to GAPDH (Mm99999915_g1). Student's $t$ test was performed for qPCR results comparing WT and 5xFAD animals.

For NanoString nCounter analysis, the neuroinflammation panel was used (catalog numbers XTCSO-MNROI1-12, Seattle, WA, USA). Two hundred nanograms of RNA was loaded per 1-month-old male mouse from the WT and PLCG2-inactivation mouse samples $(n=3)$ and was hybridized with probes for $16 \mathrm{~h}$ at $65^{\circ} \mathrm{C}$. The results obtained from the nCounter MAX Analysis System (NanoString Technologies, catalog \#NCT-SYST-LS, Seattle WA) were imported into the nSolver Analysis Software (v4.0; NanoString Technologies) for QC verification, normalization, and data statistical analysis using Advanced Analysis software (v2.0.115; NanoString Technologies). All assays were performed according to the manufacturer's protocols [29].

\section{Immunofluorescence}

The brains from mice at 8 months of age were fixed in $4 \%$ PFA overnight at $4^{\circ} \mathrm{C}$. Following overnight fixation, the brains were cryoprotected in $30 \%$ sucrose at $4^{\circ} \mathrm{C}$ and were embedded. The brains were processed on a microtome as $30-\mu \mathrm{m}$ free-floating sections. For immunostaining, at least three matched brain sections were used. Free-floating sections were washed and permeabilized in $0.1 \%$ TritonX in PBS (PBST), followed by antigen retrieval using $1 \mathrm{x}$ Reveal Decloaker (Biocare Medical) at $85^{\circ} \mathrm{C}$ for 10 min. Sections were blocked in 5\% normal donkey serum in PBST for $1 \mathrm{~h}$ at room temperature (RT). The sections were incubated in the following primary antibodies in $5 \%$ normal donkey serum in PBST overnight at $4^{\circ} \mathrm{C}$ : IBA1 (Novus Biologicals \#NB100-1028, 1:1000); P2RY12 (AnaSpec, \#AS-55043A, 1:1000). Sections were washed and visualized using the respective species-specific AlexaFluor fluorescent antibodies (diluted 1:1000 in 5\% normal donkey serum in PBST for $1 \mathrm{~h}$ at RT). Sections were washed 3 times in PBST for $10 \mathrm{~min}$, then incubated in DAPI for $5 \mathrm{~min}$. Sections were counterstained and mounted onto slides. Images were acquired on a fluorescence microscope with similar exposure and gains across stains and animals. Microglia-morphology was measured as previously described [30] using a Nikon A1R confocal microscope (Nikon Instruments, Melville, NY). Z-stacks ( $1 \mu \mathrm{m}$ steps) were acquired through layer $\mathrm{V}$ of the visual cortex in 5 slices per animal. Images were blinded, and the area coverage of microglia was calculated using ImageJ software [31]. 

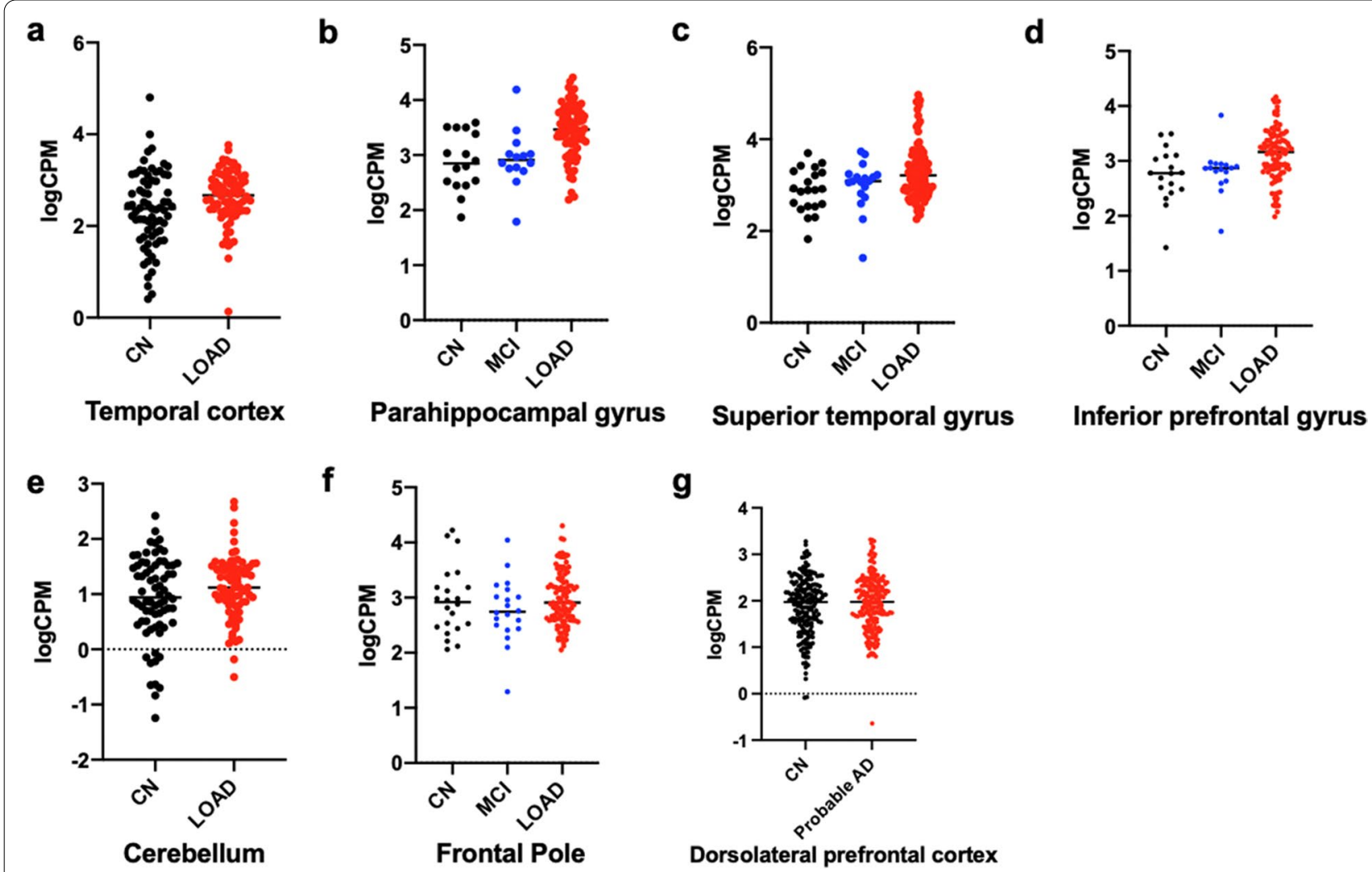

Fig. 1 Difference of PLCG2 expression levels across the diagnostic groups. Gene expression levels are shown as logCPM values. a Temporal cortex - Mayo, b Parahippocampal gyrus - MSBB, c Superior temporal gyrus - MSBB, d Inferior prefrontal gyrus - MSBB, e Cerebellum - Mayo, f Frontal pole - MSBB, and $\mathbf{g}$ Dorsolateral prefrontal cortex - ROS/MAP. CN cognitively normal older adults, AD Alzheimer's disease, MCI mild cognitive impairment

\section{Statistical analysis}

In the mouse study, statistical analyses were performed using GraphPad Prism (Version 8.4.2). Experiments at the 2-, 4-, 6- and 8-month time points were performed independently, and statistical comparisons between WT and 5xFAD AD mice were performed by an unpaired $t$ test.

\section{Results}

\section{PLCG2 expression levels are increased in LOAD, compared} to $\mathrm{CN}$

The demographic information of participants included in this study is summarized in Table 1. From gene expression analysis with age and sex as covariates, using RNA-Seq data generated from seven brain regions (Additional file 1: Table S1), we found that PLCG2 was overexpressed in LOAD patients in the temporal cortex $(\log \mathrm{FC}=0.27, p=4.56 \mathrm{E}-02$; Fig. 1a), parahippocampal gyrus $(\log \mathrm{FC}=0.55, p=1.74 \mathrm{E}-03$; Fig. $1 \mathrm{~b})$, superior temporal gyrus $(\log \mathrm{FC}=0.46, \mathrm{p}=2.55 \mathrm{E}-02$; Fig. $1 \mathrm{c})$, and inferior prefrontal gyrus $(\log \mathrm{FC}=0.36, p=1.38 \mathrm{E}-02$; Fig 1d) (Table 2). However, we did not find any diagnostic group differences in the cerebellum (Fig. 1e), frontal pole (Fig. 1f), and dorsolateral prefrontal cortex (Fig. 1g) (Table 2). With inclusion of APOE $\varepsilon 4$ carrier status as an additional covariate, PLCG2 remained overexpressed in LOAD patients in the parahippocampal gyrus $(\log \mathrm{FC}=0.57, p=2.02 \mathrm{E}-03)$, superior temporal gyrus $(\log \mathrm{FC}=0.42, p=4.95 \mathrm{E}-02)$, and inferior prefrontal cortex $(\log \mathrm{FC}=0.33, p=2.60 \mathrm{E}-02)$ (Table 2$)$. In the parahippocampal gyrus, superior temporal gyrus, and inferior prefrontal gyrus, PLCG2 was overexpressed in LOAD patients with and without $A P O E \& 4$ carrier status as an additional covariate. We then investigated whether expression levels of PLCG2 were associated with expression levels of microglia-specific marker genes (AIF1 and TMEM119). Our analysis revealed that AIF1 and TMEM119 expression levels were significantly associated with PLCG2 expression in the frontal pole (AIF1: $\beta=0.258, \mathrm{p}=1.09 \mathrm{E}-06$; TMEM119: $\beta=0.518, p=1.28 \mathrm{E}-15)$, superior temporal gyrus (AIF1: $\beta=0.33, p=8.98 \mathrm{E}-07 ;$ TMEM119: $\beta=0.71, p<2 \mathrm{E}-16)$, parahippocampal gyrus (AIF1: $\beta=0.419, p=5.43 \mathrm{E}-07$; TMEM119: $\beta=0.806, p<2 \mathrm{E}-16)$, and inferior prefrontal gyrus (AIF1: $\beta=0.257, \quad p=6.15 \mathrm{E}-06 ; \quad T M E M 119$ : $\beta=0.582, p=6.64 \mathrm{E}-15$ ) (Table 3). 
Table 2 shows the $p$-values from gene expression analysis using RNA-Seq data from the AMP-AD consortium

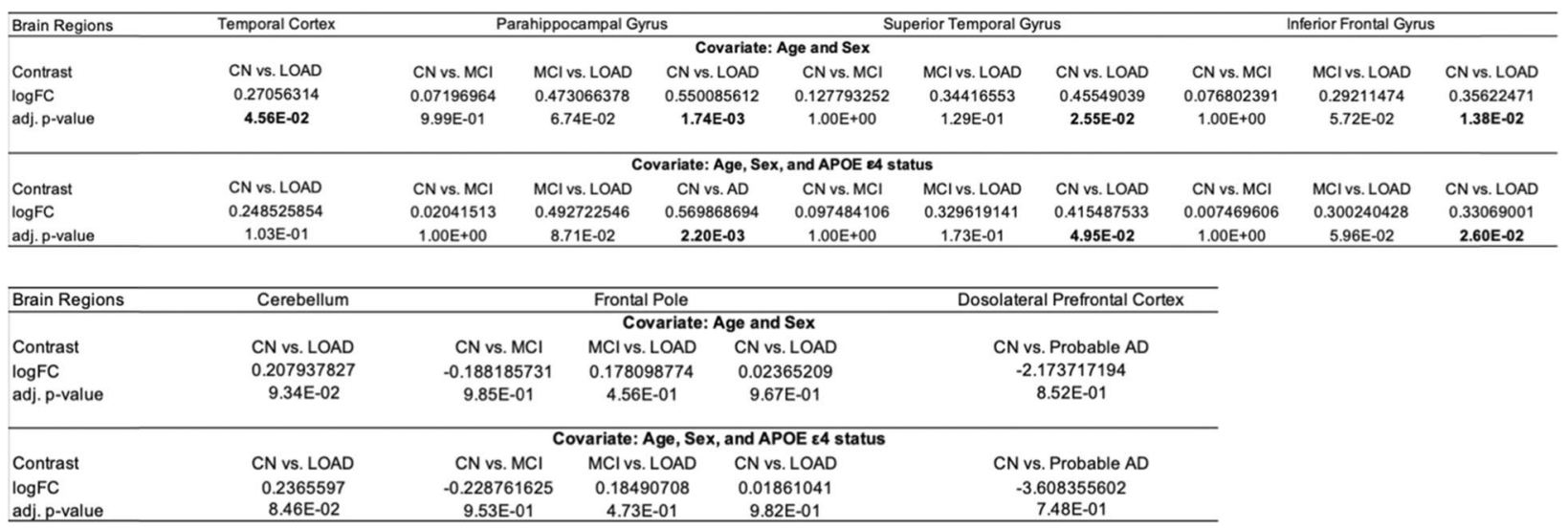

TCX temporal cortex, PHG parahippocampal gyrus, STG superior temporal gyrus, IFG inferior temporal gyrus, FP frontal pole, CER cerebellum, DLPFC dorsolateral prefrontal cortex, CN cognitively normal, AD Alzheimer's disease, MCI mild cognitive impairment, logFClog fold-change

Table 3 PLCG2 expression levels were associated with amyloid plaque density and expression levels of microglia specific markers Table 3 shows the $\beta$ coefficient $(\beta)$, standard error (SE), and $p$-value for the association analysis between PLCG2 expression levels and amyloid plaque density or expression levels of microglia specific markers, AIF1 and TMEM119 by general linear models

\begin{tabular}{|c|c|c|c|c|c|c|c|c|c|c|c|c|}
\hline \multirow{2}{*}{ Brain Regions (MSBB) } & \multicolumn{3}{|c|}{ a. Parahippocampal Gyrus } & \multicolumn{3}{|c|}{ b. Superior Temporal Gyrus } & \multicolumn{3}{|c|}{ c. Inferior Frontal Gyrus } & \multicolumn{3}{|c|}{ d. Frontal Pole } \\
\hline & $\beta$ & SE & adj. $p$-value & $\beta$ & SE & adj. $p$-value & $\beta$ & SE & adj. $p$-value & $\beta$ & SE & adj. $p$-value \\
\hline Plaque $\mathrm{Me}$ & 0.027 & 0.006 & 5.22E-05 & 0.026 & 0.006 & $2.02 \mathrm{E}-04$ & 0.017 & 0.006 & 2.73E-03 & 0.01 & 0.005 & $8.45 \mathrm{E}-02$ \\
\hline AlF1 & 0.419 & 0.078 & 5.43E-07 & 0.33 & 0.063 & $8.98 \mathrm{E}-07$ & 0.257 & 0.054 & $6.15 \mathrm{E}-06$ & 0.258 & 0.051 & $1.09 \mathrm{E}-06$ \\
\hline TMEM119 & 0.806 & 0.066 & $<2 \mathrm{E}-16$ & 0.71 & 0.064 & $<2 \mathrm{E}-16$ & 0.582 & 0.065 & $6.64 \mathrm{E}-15$ & 0.518 & 0.057 & $1.28 \mathrm{E}-15$ \\
\hline
\end{tabular}

\section{Increased PLCG2 expression levels are associated with amyloid plaques in the human brain}

We performed an association analysis of expression levels of PLCG2 and mean amyloid plaque densities measured in four brain regions. The $p$-values and beta values were obtained by linear regression in each separate brain region. Expression levels of PLCG2 in the human brain were associated with amyloid plaques in three brain regions (Table 3 and Additional file 1: Table S2). Increased PLCG2 expression levels were associated with increased amyloid plaque levels in the parahippocampal gyrus $(\beta=0.027, p=5.22 \mathrm{E}-05$; Fig. $2 \mathrm{a})$, superior temporal gyrus $(\beta=0.026, p=2.02 \mathrm{E}-04$; Fig. $2 \mathrm{~b})$, and inferior prefrontal cortex $(\beta=0.017, p=2.73 \mathrm{E}-03$; Fig. $2 \mathrm{c})$, but not in the frontal pole $(\beta=0.01, p=0.08$; Fig. $2 \mathrm{~d})$.

\section{PIcg2 expression is increased exclusively in microglia in the brains of 5xFAD mice}

Plcg2 expression was increased in the 5xFAD mouse model of amyloid pathology, consistent with our findings in human LOAD. In 5xFAD mice, Plcg2 expression was increased in the cortex (Fig. 3a) and hippocampus (Fig. 3b) of 4-, 6-, and 8-month-old mice (4-month: 1.39 -fold in the cortex and 1.37-fold in the hippocampus; 6-month: 2.37-fold in the cortex and 1.96-fold in the hippocampus; and 8-month: 2.43-fold in the cortex and 2.67-fold in the hippocampus) (Fig. 3). Furthermore, our analysis showed a disease progression-dependent increase in $\mathrm{Plcg} 2$ expression in mice with amyloid pathology. To assess our findings that PLCG2 expression levels were associated with expression levels of microglia-specific marker genes in the human brain (Table 3 and Additional file 1: Table S2), we depleted microglia in 4-month-old 5xFAD mice by treating the animals with the PLX5622 (PLX), a colony-stimulating factor receptor-1 antagonist, for 28 days [22, 28]. In the cortex, the increase in Plcg2 expression in 5xFAD mice was reduced to control levels by the PLX treatment, despite only $70 \%$ microglial depletion (Fig. 3c). Furthermore, expression levels of $\mathrm{Plcg} 2$ were restored after switching the mouse diet from the PLX diet to a normal diet for 28 further days (Fig. 3d). However, we did not observe any increase in Plcg2 expression in a 12-month tauopathy mouse model (P301S), which exhibits robust microgliosis (Additional file 2: Fig. S1).

Differential expression analysis in cognitively normal older adults and Plcg2-inactivated mouse model identified inflammation-related pathways

We performed differential expression analysis of bulk RNA-Seq data from the ROS/MAP cohort by comparing $\mathrm{CN}$ in the 25th percentile of PLCG2 gene 

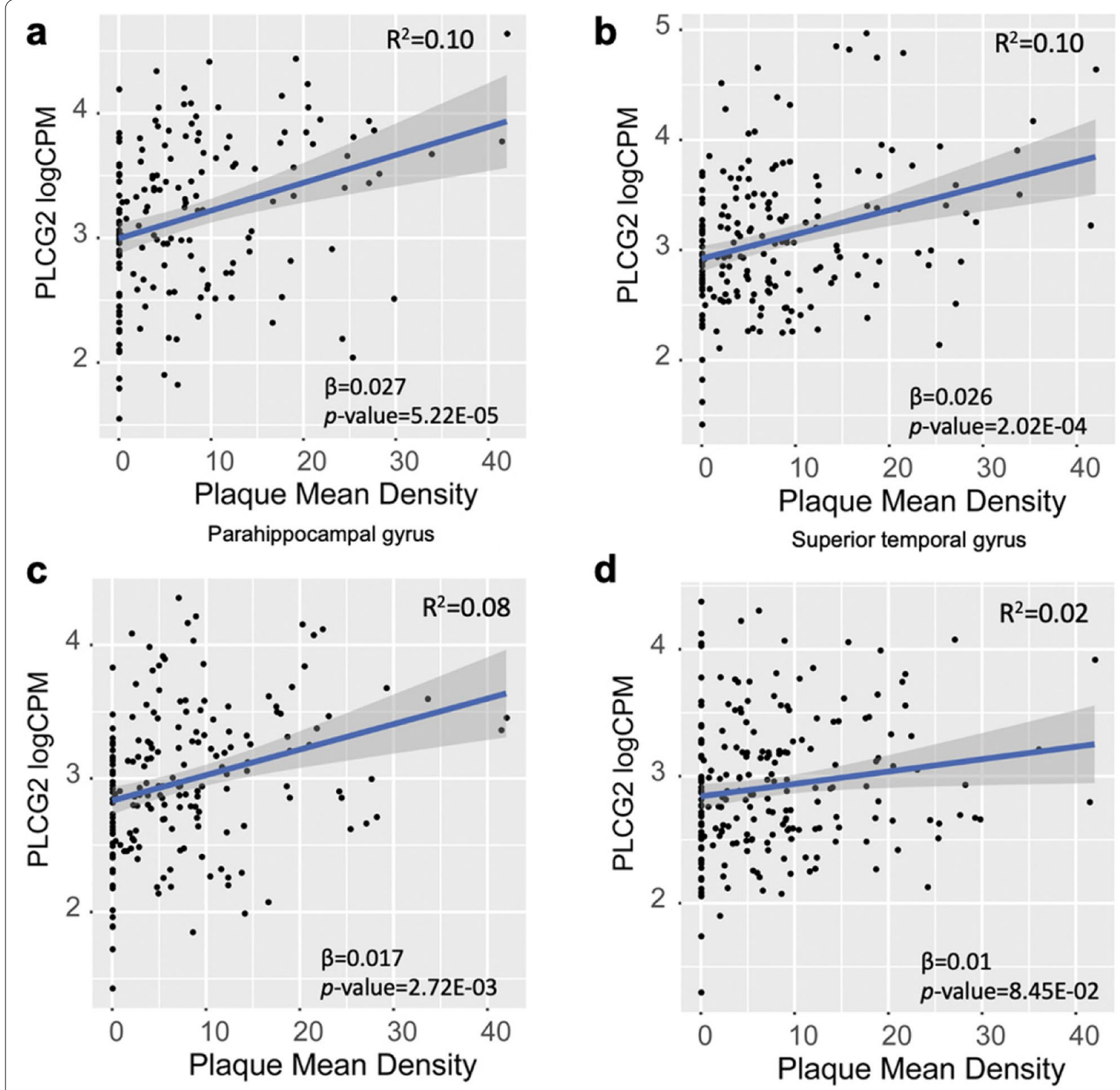

Inferior prefrontal gyrus

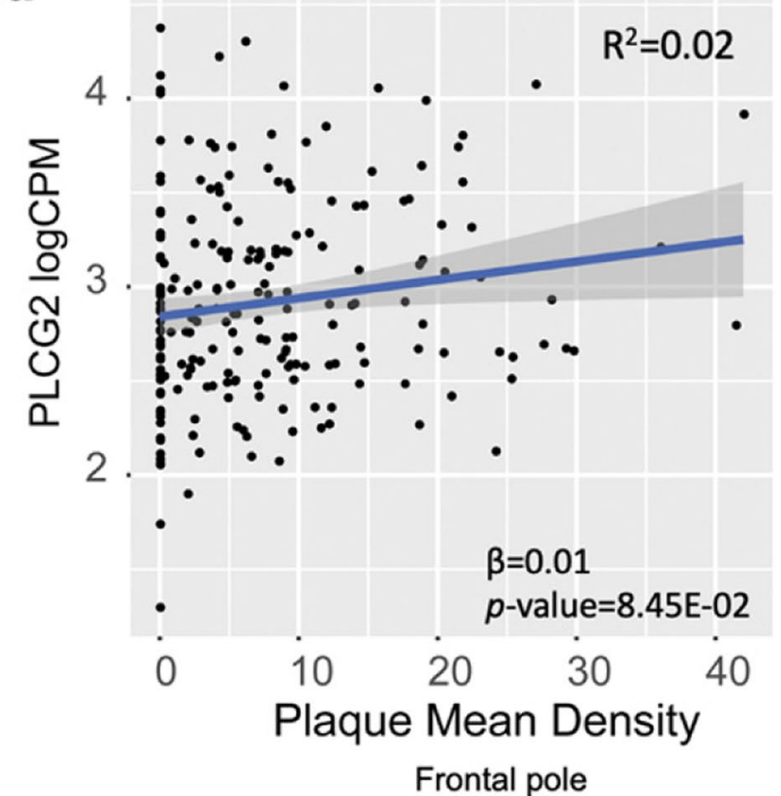

Fig. 2 Associations of PLCG2 expression levels with mean amyloid plaque density. The scatter plots show positive associations between PLCG2 expression levels and mean plaque density in the a parahippocampal gyrus, $\mathbf{b}$ superior temporal gyrus, and $\mathbf{c}$ inferior prefrontal gyrus and $\mathbf{d}$ frontal pole from the MSBB cohort

expression levels with $\mathrm{CN}$ participants in the 75th percentile of PLCG2 gene expression levels (Additional file 1: Table S3). The analysis identified 5047 significantly differentially expressed genes (FDR-corrected $p<0.05$ ), including 4566 downregulated (992 genes with larger than 1.5-fold changes) and 481 upregulated genes (6 genes with larger than 1.5-fold changes) (Fig. 4a). Pathway analysis identified several GO terms, including the inflammatory response (FDRcorrected $p=5.77 \mathrm{E}-32$ ) (Fig. $4 \mathrm{~b}$ ). To validate our findings in humans, we generated mice with inactivated Plcg2 and performed differential expression analysis by comparing Plcg2-inactivated mice with WT mice at one month of age using gene expression data from the NanoString Neuroinflammation panel (757 genes) (Additional file 1: Table S4). Our analysis identified 


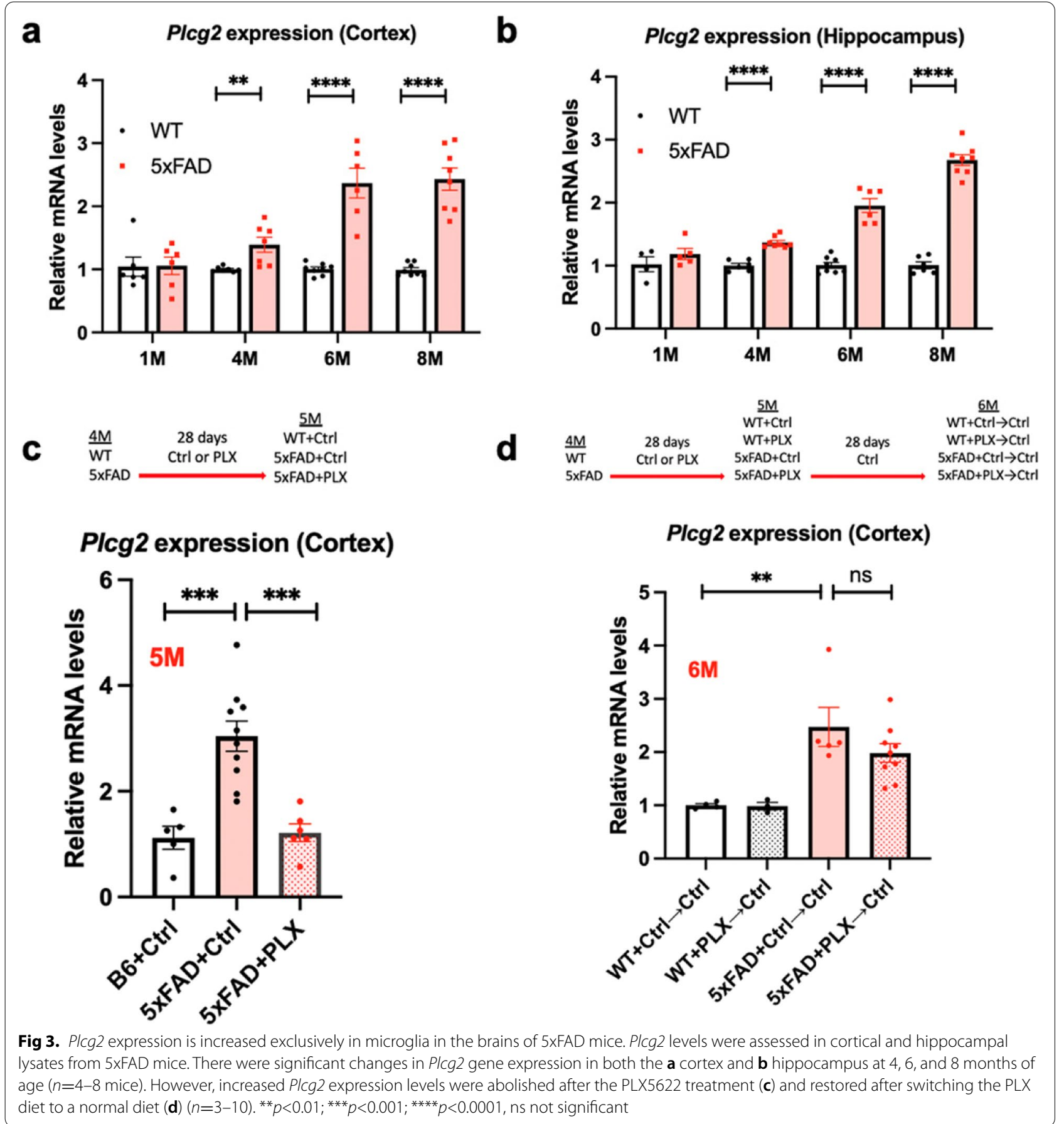

74 significantly differentially expressed genes, including 39 downregulated genes (35 genes with larger than 1.5 -fold changes) and 35 upregulated genes (14 genes with larger than 1.5 -fold changes). Of these genes, 17 genes shown in the volcano plot (16 downregulated and 1 upregulated) were identified to have the same differentially expressed directions in both humans and mice (Fig. 4c). Differentially expressed genes (FDR-corrected $p<0.05)$, including $c c l 2, c x 3 c l 1$, and $c x c l 9$, are shown in the heatmap with enrichment analysis of KEGG pathways (Additional file 2: Fig. S2a) and Gene Ontology terms for biological processes (Additional file 2: Fig. S2b), suggesting the role of PLCG2 in the inflammatory response. To determine if the differentially expressed genes/pathways were altered by microglial cell coverage or morphology, we performed immunostaining. 


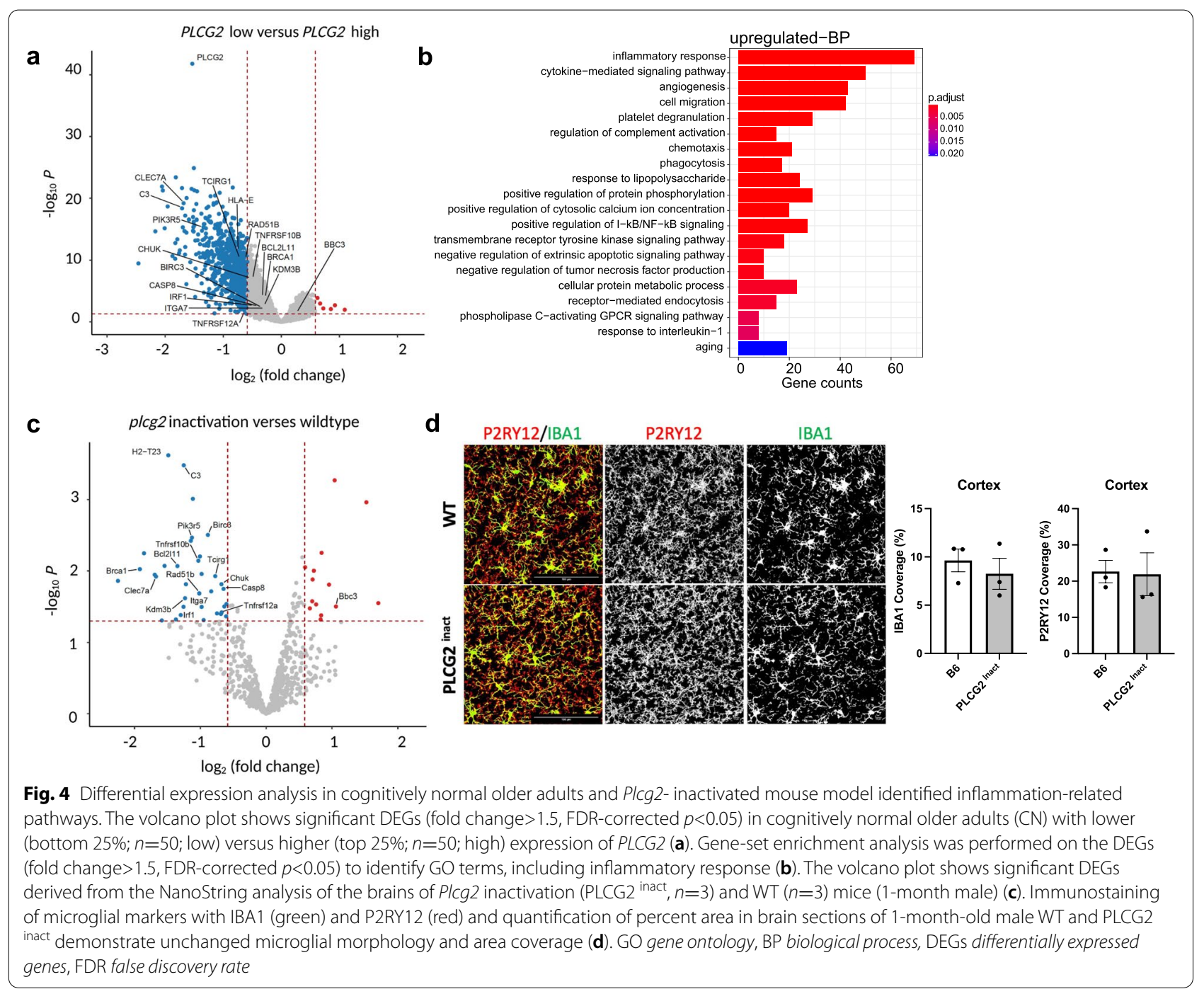

Immunostaining of 1-month-old WT and PLCG2 inact mice revealed that the microglial cell coverage and morphology were unchanged (Fig. 4d).

\section{PLCG2 co-expression network analysis of microglial single-cell RNA-Seq data identified inflammatory response-related pathways}

We analyzed single-cell RNA-Seq data from 15,021 freshly isolated microglial cells [24]. The visualization of the cells in a UMAP plot showed the separation of the cell expressed PLCG2 (Fig. 5a). PLCG2 co-expression network analysis of gene expression profiles using GENIE3 identified the co-occurrence of genes and pathways associated with PLCG2 in the microglia of MCI and AD patients (Additional file 1: Table S5). Figure 5b shows the top 20 genes that were co-expressed with PLCG2. Gene-set enrichment analysis of the top 20\% of upregulated genes co-expressed with PLCG2 (413 genes) identified significant GO terms, including response to unfolded protein and lipopolysaccharide, regulation of small GTPase-mediated signal transduction, regulation of I-kappaB kinase/NF-kappaB signaling, and regulation of cell population proliferation, as shown in Fig. 5c.

\section{Discussion}

Although PLCG2 variants were previously identified to be associated with AD risk [9, 32, 33], the role of PLCG2 in $\mathrm{AD}$ is still poorly understood. Here, we report that PLCG2 expression is increased in several brain regions of LOAD patients and that increased PLCG2 expression levels positively correlate with both brain amyloid plaques and expression levels of microglial marker genes (AIF1 and TMEM119) (Fig. 6) [34-36]. These results highlight an important relationship between amyloid plaques and PLCG2 expression, further supported by increased expression levels of Plcg2 throughout the 


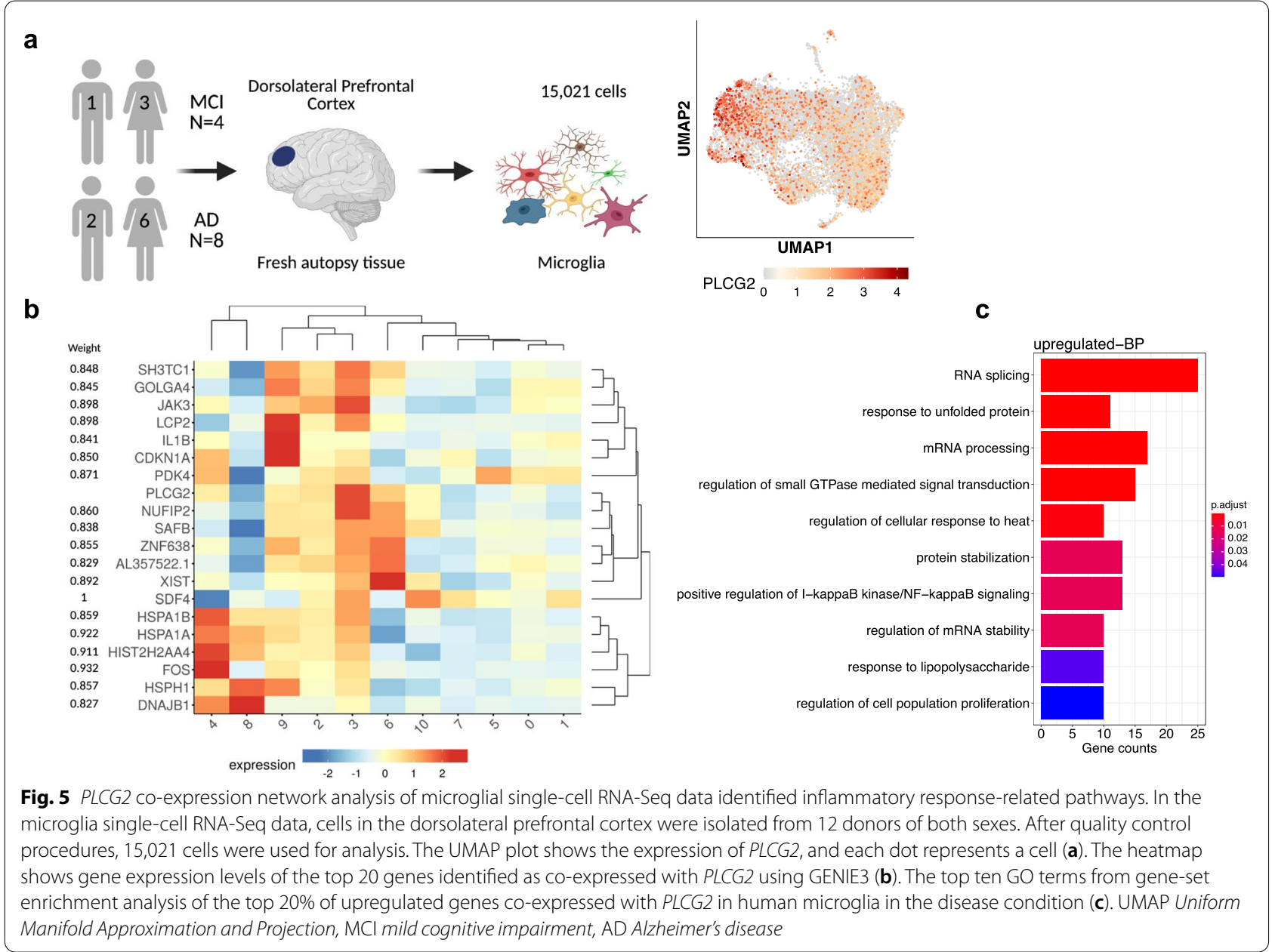

disease progression in 5xFAD mice, a well-studied model of amyloid pathology [37]. Moreover, expression levels of PLCG2 were significantly associated with expression levels of microglia-specific marker genes (AIF1 and TMEM119), which we validated in 5xFAD mice, showing that increased Plcg2 expression was induced by amyloid plaques and the increase reverted to control levels after significantly reducing microglia with the colony-stimulating factor-1 antagonist PLX5622. Interestingly, we did not observe an increase in $\mathrm{Plcg} 2$ expression in a 12-month tauopathy mouse model (P301S), which exhibits robust microgliosis, suggesting that the increase of Plcg2 might result from an association between microglia and amyloid plaques, rather than microgliosis.

Accumulating evidence suggests that the modulation of the TREM2/PLCG2 signaling pathway is a potential therapeutic candidate for AD [16]; however, the role of PLCG2 and the signaling networks associated with PLCG2 in the brain remain unclear. We identified differentially expressed genes and pathways by performing differential gene expression analysis on bulk RNASeq data from CN with 25th-percentile PLCG2 gene expression levels (low) and 75th-percentile PLCG2 gene expression levels (high). These genes and pathways are related to the inflammatory response, cytokine-mediated signaling, regulation of complement activation, chemotaxis, response to lipopolysaccharide, regulation of I-kappaB/NF-kappaB signaling, and response to interleukin-1. A previous study of human induced pluripotent stem cell-derived microglia-like (iMG) cells revealed that PLCG2 is required to induce proinflammatory signaling, a finding recapitulated by the results of our pathway analysis [38], reinforcing the role of PLCG2 in the inflammatory response. Our findings in humans were validated in mice with inactivated Plcg2 expression (PLCG2 inact), which reduced expression levels of $\mathrm{AD}$-associated microglial genes $[39,40]$, including CLEC7A, C3, and PIK3R5, in comparison with expression levels of these genes in WT mice. Importantly, the reduction of the expression 


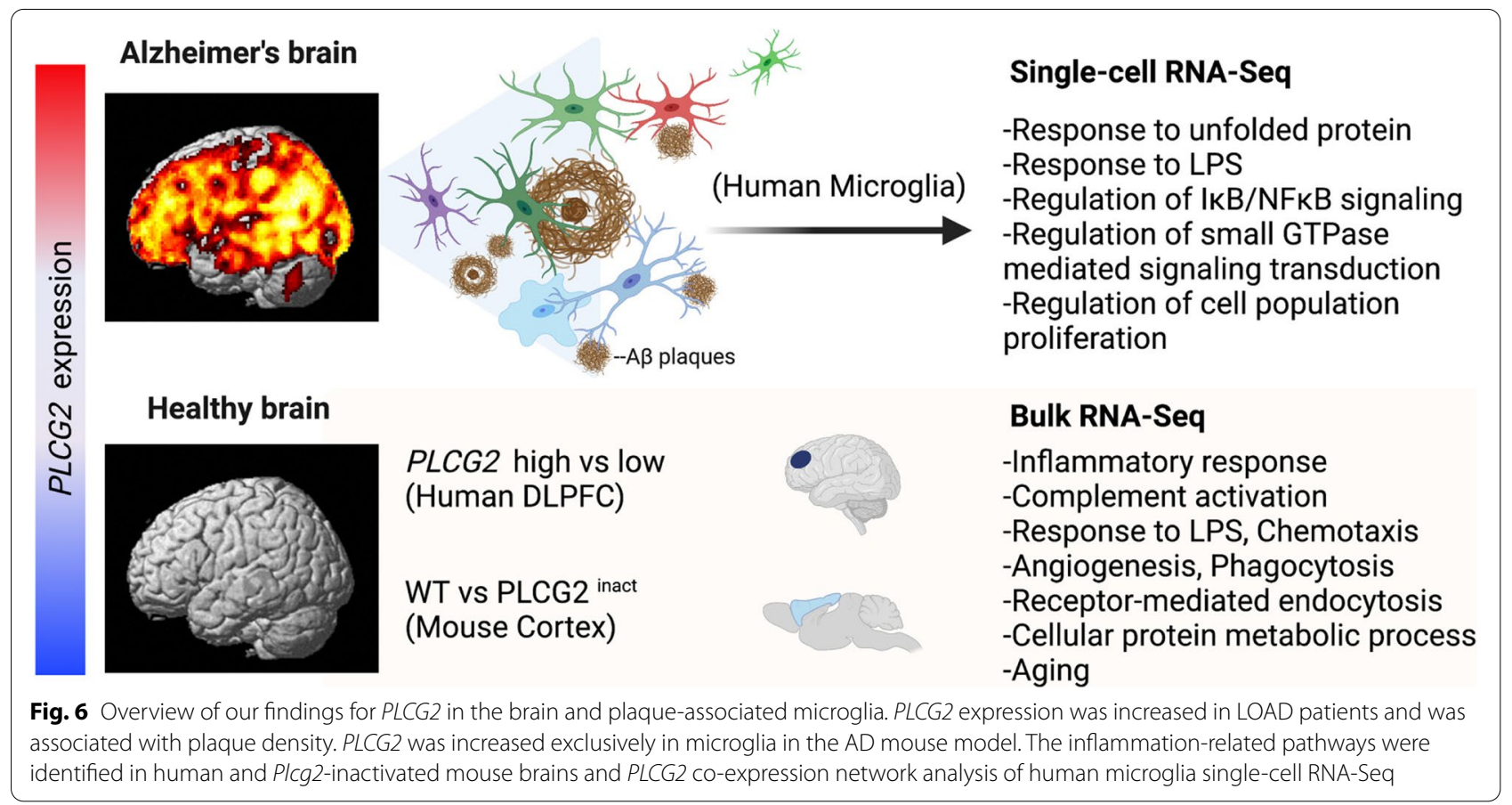

of AD-associated microglial genes was not accompanied by changes in microglial cell coverage or morphology as determined by immunostaining, indicating that the observed gene expression changes were not due to the increased numbers of microglia cells. These results suggest a potential role for PLCG2 in inflammation-related pathways.

A previous single-cell RNA-sequencing study of human $\mathrm{CN}, \mathrm{MCI}$, and $\mathrm{AD}$ microglia identified that about $80 \%$ of all microglia fell into a homeostatic subtype [24]. We performed a co-expression network analysis using microglia single-cell RNA-Seq data with fresh isolated cells from $\mathrm{MCI}$ and $\mathrm{AD}$ donors to identify working partners of PLCG2 under disease conditions that may have potential roles as microgliadirected therapeutic targets. This analysis identified $I L 1 B$ as one of the top 20 genes included in the PLCG2 co-expression network, recapitulating the result of a previous study in which $I L 1 B$ expression was downregulated in PLCG2 knock-out iMG cells after zymosan challenge [38]. Furthermore, we performed a gene-set enrichment analysis of the top 20\% of genes included in the PLCG2 co-expression network. The identified pathways were related to the response to unfolded protein, regulation of small GTPase mediated signal transduction, regulation of I-kappaB kinase/NF-kap$\mathrm{paB}$ signaling, response to lipopolysaccharide, and regulation of cell population proliferation. These findings suggest a potential role for PLCG2 in AD microglia, which may allow us to develop a novel therapeutic strategy targeting PLCG2 or the signaling networks associated with it.

\section{Conclusions}

In conclusion, PLCG2 expression is increased in several brain regions in LOAD patients and significantly correlates with brain amyloid burden in LOAD patients and AD model mice. Specifically, 5xFAD amyloid pathology mice, we observed a disease progression-dependent increase in Plcg2 expression. The results of this study show that 5xFAD and Plcg2-inactivation mouse models are appropriate for studying PLCG2 in AD. We believe that future studies of genetic mouse models are needed to further clarify the role of PLCG2 in plaque-associated microglia and to determine whether decreased PLCG2 expression in plaque-associated microglia favors disease exacerbation or attenuation. Moreover, investigating how PLCG2 modulates microglia phenotypes in AD may identify novel therapeutic strategies for microglia-targeted AD therapies. 


\section{Supplementary Information}

The online version contains supplementary material available at https://doi. org/10.1186/s13073-022-01022-0.

Additional file 1: Table S1: PLCG2 expression levels in different brain regions. Table S2: Levels of plaque mean density, and the expression levels of PLCG2, AIF1, and TMEM119 in MSBB cohort. Table S3: Differential expression analysis from CN with $25^{\text {th }}$-percentile PLCG2 gene expression levels and $75^{\text {th }}$-percentile PLCG2 gene expression levels. Table S4: Differential expression analysis of mouse data. Table S5: Co-expression analysis of PLCG2 in human microglia scRNA-seq analysis.

Additional file 2: Figure S1: Plcg2 expression does not change in a 12-month tauopathy mouse model, despite microgliosis. Figure S2 Differential expression analysis in Plcg2 inactivation mice identified inflammation-related pathways.

\section{Acknowledgements}

We thank Louise Pay for her critical comments on the manuscript. We thank Juan Francisco Codocedo, Kathryn P. MacPherson, Shweta S. Puntambekar, Vaishnavi Sunil Jadhav for helpful discussions, and Guixiang Xu, Roxanne Y. Williams, and Teaya N. Thomas for their help with taking care of the mice and genotyping. Figure 6 was generated using Biorender.

\section{Authors' contributions}

A.P.T, C.D, A.L.O, Y.L, G.E.L, B.T.L, S.J.B, and K.N designed the study. A.P.T, C.D, M.M, P.B.L, E.J.M, B.T.C, and K.N performed the experiments and analyzed the data. A.P.T, G.E.L, S.J.B, and K.N wrote the manuscript. All authors discussed the results and commented on the manuscript. The authors read and approved the final manuscript.

\section{Funding}

This project was supported by the Stark Neurosciences Research Institute, the Indiana Alzheimer Disease Center, Eli Lilly and Company, and by the Indiana Clinical and Translational Sciences Institute, funded in part by grant \# UL1TR002529 from the National Institutes of Health, National Center for Advancing Translational Sciences. The content is solely the responsibility of the authors and does not necessarily represent the official views of the National Institutes of Health. The stipend for A.P.T. was provided in part through a fellowship funded by Eli Lilly and Company. In addition, this work was supported by NIA grant RF1 AG051495 (B.T.L and G.E.L), NIA grant RF1 AG050597 (G.E.L), NIA grant U54 AG054345 (B.T.L et al.), NIA grant K01 AG054753 (A.L.O), NIA grant RF1 AG074566 (G.E.L, B.T.L, and S.J.B), NIA grant R03 AG063250 (K.N), and NIH grant NLM R01 LM012535 (K.N).

\section{Availability of data and materials}

RNA-Seq data were downloaded from the Accelerating Medicines Partnership for Alzheimer's Disease (AMP-AD) Consortium (https://adknowledgeport al.synapse.org/Explore/Programs/DetailsPage?Program=AMP-AD): The raw data from ROS/MAP cohort (https://www.synapse.org/\#!Synapse:syn8456638) [17], Mayo cohort (https://www.synapse.org/\#!!Synapse:syn8466816) [18], and MSBB cohort (https://www.synapse.org/\#!Synapse:syn8485017) [19] are accessible through the Synapse database (https://www.synapse.org/). The raw data from Olah et al. are accessible through Synapse (https://www.synapse. org/\#!Synapse:Syn21438358) [24]. The NanoString data reported in this paper are available in the Gene Expression Omnibus (GEO) database with accession ID GSE195650, https://www.ncbi.nlm.nih.gov/geo/query/acc.cgi?acc=GSE19 5650 [41]. Our single-cell-based transcriptomic data analysis from human microglia are available in the form of a browsable platform at https://andyp tsai.shinyapps.io/ROSMAP_micorglia_scRNAseq_explorer/.

\section{Declarations}

\section{Ethics approval and consent to participate}

All experimental procedures and animal care were approved by the Institutional American Association for Laboratory Animal Science Care and Use Committee (IACUC, protocol\# 20007-MD/R/HZ and 18099-MD/R/HZ/E/AR) and performed in accordance with the Stark Neurosciences Research Institute
Laboratory Animal Resource Center (LARC), Indiana University School of Medicine.

\section{Consent for publication}

Not applicable.

\section{Competing interests}

The authors declare that they have no competing interests. The stipend for A.P.T. was provided in part through a fellowship funded by Eli Lilly and Company; however, the project was not sponsored by Eli Lilly and Company and they had no influence on the design, conduct, or reporting of the research.

\section{Author details}

${ }^{1}$ Stark Neurosciences Research Institute, Indiana University School of Medicine, Indianapolis, IN, USA. ${ }^{2}$ Department of Medical and Molecular Genetics, Center for Computational Biology and Bioinformatics, Indiana University School of Medicine, Indianapolis, IN, USA. ${ }^{3}$ Northeast Ohio Medical University, Rootstown, OH, USA. ${ }^{4}$ Department of Radiology \& Imaging Sciences, Indiana University School of Medicine, Indianapolis, IN, USA. ${ }^{5}$ Department of Medical and Molecular Genetics, Indiana University School of Medicine, Indianapolis, IN, USA. ${ }^{6}$ Department of Anatomy and Cell Biology, Indiana University School of Medicine, Indianapolis, IN, USA.

Received: 9 August 2021 Accepted: 7 February 2022

Published online: 18 February 2022

\section{References}

1. Isik AT. Late onset Alzheimer's disease in older people. Clin Interv Aging 2010;5:307-11.

2. Riedel BC, Thompson PM, Brinton RD. Age, APOE and sex: triad of risk of Alzheimer's disease. J Steroid Biochem Mol Biol. 2016;160:134-47.

3. Reiman EM, Arboleda-Velasquez JF, Quiroz YT, Huentelman MJ, Beach TG, Caselli RJ, et al. Exceptionally low likelihood of Alzheimer's dementia in APOE2 homozygotes from a 5,000-person neuropathological study. Nat Commun. 2020;11:667.

4. Kim J, Basak JM, Holtzman DM. The role of apolipoprotein E in Alzheimer's disease. Neuron. 2009:63:287-303.

5. Corrada MM, Brookmeyer R, Paganini-Hill A, Berlau D, Kawas CH. Dementia incidence continues to increase with age in the oldest old: the $90+$ study. Ann Neurol. 2010;67:114-21.

6. Kunkle BW, Grenier-Boley B, Sims R, Bis JC, Damotte V, Naj AC, et al. Genetic meta-analysis of diagnosed Alzheimer's disease identifies new risk loci and implicates Abeta, tau, immunity and lipid processing. Nat Genet. 2019:51:414-30.

7. Efthymiou AG, Goate AM. Late onset Alzheimer's disease genetics implicates microglial pathways in disease risk. Mol Neurodegener. 2017;12:43.

8. Karch CM, Goate AM. Alzheimer's disease risk genes and mechanisms of disease pathogenesis. Biol Psychiatry. 2015;77:43-51.

9. Sims R, van der Lee SJ, Naj AC, Bellenguez C, Badarinarayan N, Jakobsdottir J, et al. Rare coding variants in PLCG2, ABI3, and TREM2 implicate microglial-mediated innate immunity in Alzheimer's disease. Nat Genet. 2017;49:1373-84

10. Magno L, Lessard CB, Martins M, Lang V, Cruz P, Asi Y, et al. Alzheimer's disease phospholipase C-gamma-2 (PLCG2) protective variant is a functional hypermorph. Alzheimers Res Ther. 2019;11:16.

11. Maguire E, Menzies GE, Phillips T, Sasner M, Williams HM, Czubala MA, et al. PIP2 depletion and altered endocytosis caused by expression of Alzheimer's disease-protective variant PLCgamma2 R522. EMBO J. 2021:40:e105603.

12. Hernandez D, Egan SE, Yulug IG, Fisher EM. Mapping the gene that encodes phosphatidylinositol-specific phospholipase C-gamma 2 in the human and the mouse. Genomics. 1994;23:504-7.

13. Takalo M, Wittrahm R, Wefers B, Parhizkar S, Jokivarsi K, Kuulasmaa T, et al. The Alzheimer's disease-associated protective Plcy2-P522R variant promotes immune functions. Mol Neurodegener. 2020;15:52.

14. Hajicek N, Keith NC, Siraliev-Perez E, Temple BR, Huang W, Zhang Q Harden TK, Sondek J. Structural basis for the activation of PLC-gamma isozymes by phosphorylation and cancer-associated mutations. Elife. 2019;8. 
15. Andreone BJ, Przybyla L, Llapashtica C, Rana A, Davis SS, van Lengerich B, et al. Alzheimer's-associated PLCY2 is a signaling node required for both TREM2 function and the inflammatory response in human microglia. Nat Neurosci. 2020;23:927-38

16. Magno L, Bunney TD, Mead E, Svensson F, Bictash MN. TREM2/PLCY2 signalling in immune cells: function, structural insight, and potential therapeutic modulation. Mol Neurodegener. 2021;16:22.

17. De Jager PL, Ma Y, McCabe C, Xu J, Vardarajan BN, Felsky D, et al. A multiomic atlas of the human frontal cortex for aging and Alzheimer's disease research. Sci Data. 2018;5:180142.

18. Allen M, Carrasquillo MM, Funk C, Heavner BD, Zou F, Younkin CS, et al. Human whole genome genotype and transcriptome data for Alzheimer's and other neurodegenerative diseases. Sci Data. 2016;3:160089.

19. Wang M, Beckmann ND, Roussos P, Wang E, Zhou X, Wang Q, et al. The Mount Sinai cohort of large-scale genomic, transcriptomic and proteomic data in Alzheimer's disease. Sci Data. 2018:5:180185.

20. Morris JC. The Clinical Dementia Rating (CDR): current version and scoring rules. Neurology. 1993;43:2412-4.

21. Bennett DA, Buchman AS, Boyle PA, Barnes LL, Wilson RS, Schneider JA. Religious orders study and rush memory and aging project. J Alzheimers Dis. 2018;64:S161-89.

22. Tsai AP, Lin PB, Dong C, Moutinho M, Casali BT, Liu Y, et al. INPP5D expression is associated with risk for Alzheimer's disease and induced by plaque-associated microglia. Neurobiol Dis. 2021;153:105303.

23. Ritchie ME, Phipson B, Wu D, Hu Y, Law CW, Shi W, et al. limma powers differential expression analyses for RNA-sequencing and microarray studies. Nucleic Acids Res. 2015;43:e47.

24. Olah M, Menon V, Habib N, Taga MF, Ma Y, Yung CJ, et al. Single cell RNA sequencing of human microglia uncovers a subset associated with Alzheimer's disease. Nat Commun. 2020;11:6129.

25. Hao Y, Hao S, Andersen-Nissen E, Mauck WM 3rd, Zheng S, Butler A, et al. Integrated analysis of multimodal single-cell data. Cell. 2021;184(3573-3587):e3529.

26. Huynh-Thu VA, Irrthum A, Wehenkel L, Geurts P. Inferring regulatory networks from expression data using tree-based methods. PLoS One. 2010;5.

27. Yu G, Wang LG, Han Y, He QY. clusterProfiler: an R package for comparing biological themes among gene clusters. Omics. 2012;16:284-7.

28. Casali BT, MacPherson KP, Reed-Geaghan EG, Landreth GE. Microglia depletion rapidly and reversibly alters amyloid pathology by modification of plaque compaction and morphologies. Neurobiol Dis. 2020;142:104956.

29. Reilly AM, Tsai AP, Lin PB, Ericsson AC, Oblak AL, Ren H. Metabolic Defects Caused by High-Fat Diet Modify Disease Risk through Inflammatory and Amyloidogenic Pathways in a Mouse Model of Alzheimer's Disease. Nutrients. 2020;12.

30. Greve HJ, Mumaw CL, Messenger EJ, Kodavanti PRS, Royland JL, Kodavanti UP, et al. Diesel exhaust impairs TREM2 to dysregulate neuroinflammation. J Neuroinflammation. 2020;17:351

31. Schneider CA, Rasband WS, Eliceiri KW. NIH Image to ImageJ: 25 years of image analysis. Nat Methods. 2012;9:671-5.

32. Kleineidam L, Chouraki V, Prochnicki T, van der Lee SJ, Madrid-Marquez L, Wagner-Thelen H, Karaca I, Weinhold L, Wolfsgruber S, Boland A, et al. PLCG2 protective variant p.P522R modulates tau pathology and disease progression in patients with mild cognitive impairment. Acta Neuropathol. 2020.

33. Olive C, Ibanez L, Farias FHG, Wang F, Budde JP, Norton JB, et al. Examination of the effect of rare variants in TREM2, ABI3, and PLCG2 in LOAD through multiple phenotypes. J Alzheimers Dis. 2020;77:1469-82.

34. Hopperton KE, Mohammad D, Trepanier MO, Giuliano V, Bazinet RP. Markers of microglia in post-mortem brain samples from patients with Alzheimer's disease: a systematic review. Mol Psychiatry. 2018;23:177-98.

35. Kaiser T, Feng G. Tmem119-EGFP and Tmem119-CreERT2 Transgenic Mice for Labeling and Manipulating Microglia. eNeuro. 2019;6.

36. Satoh J, Kino Y, Asahina N, Takitani M, Miyoshi J, Ishida T, et al. TMEM119 marks a subset of microglia in the human brain. Neuropathology. 2016:36:39-49.

37. Everett KL, Bunney TD, Yoon Y, Rodrigues-Lima F, Harris R, Driscoll PC, et al. Characterization of phospholipase C gamma enzymes with gain-offunction mutations. J Biol Chem. 2009;284:23083-93.

38. Andreone BJ, Przybyla L, Llapashtica C, Rana A, Davis SS, van Lengerich B, et al. Alzheimer's-associated PLCgamma2 is a signaling node required for both TREM2 function and the inflammatory response in human microglia. Nat Neurosci. 2020;23:927-38.

39. Krasemann S, Madore C, Cialic R, Baufeld C, Calcagno N, El Fatimy R, et al. The TREM2-APOE pathway drives the transcriptional phenotype of dysfunctional microglia in neurodegenerative diseases. Immunity. 2017;47(566-581):e569.

40. Keren-Shaul H, Spinrad A, Weiner A, Matcovitch-Natan O, Dvir-Szternfeld $\mathrm{R}$, Ulland TK, et al. A unique microglia type associated with restricting development of Alzheimer's disease. Cell. 2017;169:1276-1290.e1217.

41. Tsai AP, Dong C, Lin PB, Messenger EJ, Casali BT, Moutinho M, Liu Y, Oblak AL, Lamb BT, Landreth GE, Bissel SJ, Nho K, et al. PLCG2 is Associated with the Inflammatory Response and is Induced by Amyloid Plaques in Alzheimer's Disease. BioProject, PRJNA801624, NCBI Sequence Read Archive 2022. https://www.ncbi.n/m.nih.gov/geo/query/acc.cgi?acc=GSE195650.

\section{Publisher's Note}

Springer Nature remains neutral with regard to jurisdictional claims in published maps and institutional affiliations.
Ready to submit your research? Choose BMC and benefit from:

- fast, convenient online submission

- thorough peer review by experienced researchers in your field

- rapid publication on acceptance

- support for research data, including large and complex data types

- gold Open Access which fosters wider collaboration and increased citations

- maximum visibility for your research: over $100 \mathrm{M}$ website views per year

At BMC, research is always in progress.

Learn more biomedcentral.com/submissions 